\title{
Using Character Recognition for Plate LOCALIZATION
}

\author{
Lama Hamandi ${ }^{1}$, Khaled Almustafa ${ }^{2}$, Rached Zantout ${ }^{3}$ and Hasan Obeid ${ }^{4}$ \\ ${ }^{1}$ Electrical and Computer Engineering Dept., \\ American University of Beirut, Beirut, Lebanon \\ Ih13 daub.edu.1b \\ ${ }^{2}$ College of Computer and Information Sciences, \\ Prince Sultan University, Riyadh, Saudi Arabia \\ kalmustafaepsu.edu.sa \\ ${ }^{3}$ Math and Computer Science Dept., \\ Beirut Arab University, Beirut, Lebanon \\ rached.zantoutegmail.com \\ ${ }^{4}$ Zawya, Beirut, Lebanon \\ hasanobeidegmail.com
}

\begin{abstract}
In this paper, the "character recognition" approach to recognizing a vehicle license plate is used for localizing Saudi license plates. The proposed algorithm filters out all possible objects from the license plate image and focuses on the resulting objects. The coordinates of the center point of the bounding box for these objects is calculated and then possible alignments between these objects are checked. After finding the aligned objects, the recognition algorithms are applied to differentiate the numbers from the letters in the plate.
\end{abstract}

\section{KEYWORDS}

License Plate Recognition, Plate Localization, Character Recognition

\section{INTRODUCTION}

Automatic License Plate Recognition (ALPR) systems process images to find license plates and recognize the plate characters. ALPR is usually divided into two major parts, localization and recognition. Localization locates the part of the image which contains the license plate, while recognition identifies the individual characters on the assumption that the image being identified is that of a license plate.

Localization algorithms can follow one of three approaches [1]. The first approach is color-based. It relies on character colors to distinguish between the characters and the background. The second approach is edge-based. It uses lines which are the edges of the characters and their boundaries. The third approach is texture-based. It locates a license plate by looking for specific patterns or textures in the image.

In this paper, localization of the plate is done through the recognition of the possible characters in the plate. Recognizing these characters is done by applying three different recognition algorithms 
The International Journal of Multimedia \& Its Applications (IJMA) Vol.4, No.5, October 2012

$[2,3,4]$. Localization is done by first filtering all possible objects from the license plate image. Then possible alignment between objects is used along with information about possibilities of letters and digits for each object to localize the Saudi license plate.

In section 2 a brief literature review is presented. In section 3, Saudi license plates are described. In section 4 the algorithm is detailed. Section 5 presents the various thresholds used and a discussion of the results. The paper is concluded in section 6 with a summary of achievements and suggestions for future research.

\section{LITERATURE REVIEW}

License plate localization in an image, is usually done using either histogram processing [5,6] (gray value variation), line detection [7] (edge detection), blob analysis [8,9] (study of regions), or a combination of these approaches. In the line processing approach, Hough Transform is used to find character lines, followed by grouping lines into closed boxes representing the characters in the license plate [7]. Histogram processing consists of extracting lines from the image (usually, either vertically or horizontally) and studying the pixel values for each of these lines. In [6] text areas on a mixed image are extracted using the numbers of white and black pixels in extracted lines. Other histogram processing methods use locations where pixels change from white to dark or vice versa to localize a license plate $[5,7]$.

In [10], low-contrast and dynamic-range problems are solved using histogram equalization. The license plate is localized using texture properties such as the aspect ratio, and color similarity.

The blob analysis approach deals with regions instead of individual pixels. Bounding boxes surrounding various regions are studied and their characteristics are used to locate license plates. By thresholding regions according to the width, height, area, width to height ratio [8], and position on the $\mathrm{x}$-axis and $\mathrm{y}$-axis $[9,11]$ of the bounding boxes, possible license plate candidates are identified.

In [12] and [13] Super-resolution reconstruction algorithms produce images of higher resolution from low resolution frame sequences and can be applied in real-time recognition systems. In [14] plate extraction is done using an image feature extraction process. Geometric features are probabilistically modeled and detected using different detection thresholds. These results are then used within the Bayesian framework to obtain features.

\section{SAUdi License Plates}

Figure 1 shows the new license plates that exist on the streets in Saudi Arabia currently. New Saudi Plates contain Arabic and English letters and Hindi and Arabic numerals. The width version of the new license plates is divided into six rectangles. The upper left rectangle contains Hindi numerals. The lower left rectangle contains Arabic numerals. The upper middle rectangle contains Arabic letters and the lower middle rectangle contains English letters. The upper right rectangle contains the logo of Saudi Arabia and the lower right rectangle contains the letters K S A written vertically along with a security symbol.

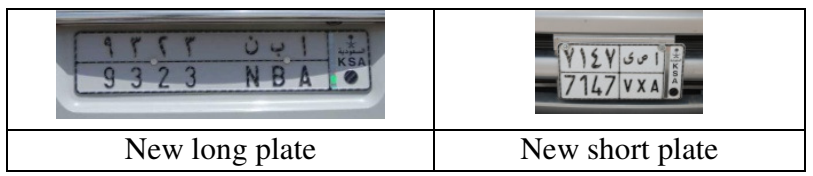

Figure 1. Long and Short Saudi Plates 
The International Journal of Multimedia \& Its Applications (IJMA) Vol.4, No.5, October 2012

The long version of the new license plate has only three rectangles inside the license plate. The upper left rectangle contains Hindi numerals to its left and Arabic letters to its right. The lower left rectangle contains English letters to the right and Arabic numerals to the left. The leftmost rectangle contains the logo of Saudi Arabia, the letters K S A written horizontally and the security symbol. Both types of new license plates are characterized by exactly three characters in the letter areas and up to four characters in the number areas. Moreover, each character in the English section corresponds to a unique character in the Arabic section. Finally, the English character is always below its corresponding Arabic character.As shown in Figure 2, in the new Saudi plates, each character, as well as the lines dividing the regions are painted with "The Kingdom of Saudi Arabia" in both Arabic and English, for authentication reasons.

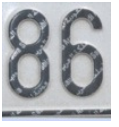

Figure 2. Printing along the characters in new plates

Table 1 shows the equivalence between English and Arabic characters in new license plates. Not all letters in the Arabic alphabet neither all characters in the English alphabet are in use in current new Saudi license plates. However, all digits (0 through 9) are in.

Table 1. Equivalence of English and Arabic Characters

\begin{tabular}{|c|c|c|c|c|c|}
\hline 1 & $\mathrm{~A}$ & ق & $\mathrm{G}$ & $r$ & 2 \\
\hline ب ب & B & ك5 & $\mathrm{K}$ & $r$ & 3 \\
\hline$\tau$ & $\mathrm{J}$ & J & $\mathrm{L}$ & $\varepsilon$ & 4 \\
\hline د & $\mathrm{D}$ & r & $\mathrm{Z}$ & 0 & 5 \\
\hline J & $\mathrm{R}$ & ن & $\mathrm{N}$ & 7 & 6 \\
\hline س & $S$ & 0 & $\mathrm{H}$ & $V$ & 7 \\
\hline ص & $\mathrm{X}$ & 9 & $\mathrm{U}$ & 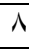 & 8 \\
\hline b & $\mathrm{T}$ & ى & $\mathrm{V}$ & 9 & 9 \\
\hline$\varepsilon$ & $\mathrm{E}$ & 1 & 1 & . & 0 \\
\hline
\end{tabular}

\section{Methodology}

The Character Recognition Algorithm for Plate Localization consists of the following steps:

- Pre-process the license plate

- Recognize all blobs/objects by filtering out very small or very large objects that cannot be candidates for numbers or characters.

- Find the $\mathrm{x}$-coordinates of objects and find vertically aligned objects.

- If two vertically aligned objects are found, verify if these objects are numbers by using the following three algorithms: 
The International Journal of Multimedia \& Its Applications (IJMA) Vol.4, No.5, October 2012

1. Pixels Density algorithm [3]

2. Position of Peaks algorithm [2].

3. Number of Peaks algorithm [4]

- Then verify if these two vertically aligned objects are letters using the same three algorithms.

The characters within the license plate might contain gaps, rough contour, small holes, or narrow breaks. Preprocessing is used to improve the image quality [15]. It converts the colored image into a binary version where pixels in the image are labeled as "object pixels" and "background pixels."

After finding two vertically aligned objects, three character recognition algorithms are run to verify that these objects are numbers. A bounding box is defined around each object. Then six lines are taken horizontally and vertically at different locations inside the bounding box of each object. The locations are defined to be at the center, the upper, lower, right, and left of the bounding box. All lines except for the center lines are taken at a factor $f$ of the height or width $(f=$ $1 / 3,1 / 4,1 / 5,1 / 10)$.

The first algorithm is the Pixels density algorithm. The algorithm calculates percentages of the object pixels along the six previously mentioned lines. The pixels densities obtained identify possible candidate characters for this object.

The second algorithm is the Position of Peaks algorithm. A peak is defined as a crossing from a black pixel to a white pixel. If a line starts with a white pixel, it is considered to have one peak at the beginning. This algorithm divides each bounding box vertically into 3 equal sections (Left, Center and Right) and horizontally into 3 sections (Top, Middle and Bottom). The peaks for each of the six strategic lines are found and their positions (L, C, R) for horizontal lines or (T, M, B) for vertical lines are determined. The positions of peaks found permit to identify candidate characters for the considered object.

The third algorithm is the Number of Peaks algorithm. This algorithm calculates the number of peaks along each of the six strategic lines. The numbers obtained permit to identify candidate characters for the object considered.

Finally, using the results of all three algorithms, and the fact that there is a correspondence between the top Arabic letter or number and the corresponding vertically aligned lower English letter or number, the object is recognized.

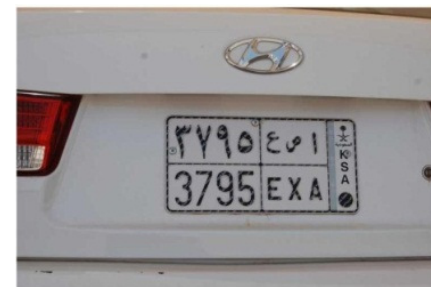

Figure 3. License Plate Used for Simulation 
The International Journal of Multimedia \& Its Applications (IJMA) Vol.4, No.5, October 2012

Figure 3 shows a license plate example and Figure 4 shows the resulting plate after preprocessing. As an example the rear light in Figure 4 will be filtered as a large object. The trunk key hole will also be filtered as a small object.

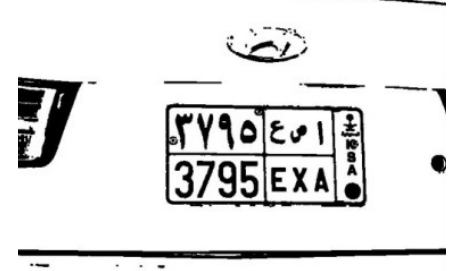

Figure 4. After Pre-Processing

The objects, from Figure 4, that made it through the filter can be seen in Figures 5, 6 and 7.

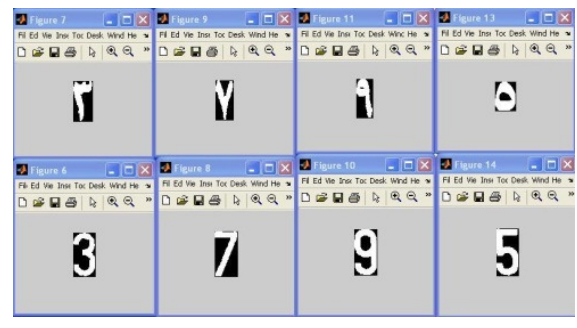

Figure 5. Objects to be considered as Numbers

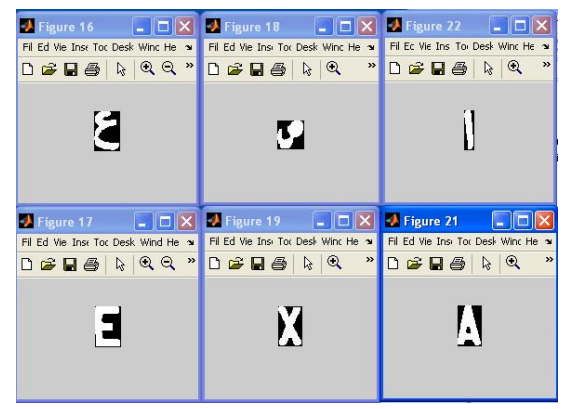

Figure 6. Objects to be considered as Letters

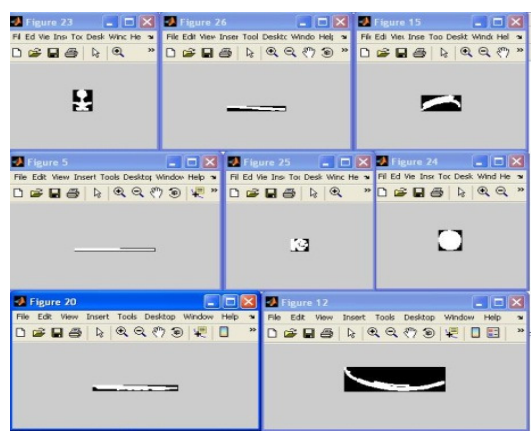

Figure 7. Unwanted Resultant Objects 
The International Journal of Multimedia \& Its Applications (IJMA) Vol.4, No.5, October 2012

The algorithm then computes the x-coordinates of the center points for each possible object as shown in Table 2 . Two objects having close $\mathrm{x}$-coordinates are vertically aligned. This means they are possible candidates for letters or numbers in the plate (Arabic on top of English). However, they might be the letters K.S.A as well as the palm tree symbol in the plate, as seen in the rightmost part of the plate in Figure 3. In Table 3 in the second and third cell of the first row, the $\mathrm{x}$ coordinates of two objects (that correspond to the number 3 in Arabic and Hindi) are almost equal (290.5 vs. 291.5) Which means that they are vertically aligned.

Table 2. X-Coordinates for all Filtered Objects

\begin{tabular}{|l|l|l|l|l|l|l|l|}
\hline 271.5 & 290.5 & 291.5 & 329.5 & 330.5 & 367.5 & 368.5 & 382.5 \\
\hline 406.5 & 407.5 & 423.5 & 459.5 & 461.5 & 495.5 & 499.5 & 519.5 \\
\hline 538.5 & 543.5 & 589.5 & 591.5 & 594.5 & 663.5 & & \\
\hline
\end{tabular}

First we check to see if the object is a number, so we run all three algorithms associated with recognizing numbers on it for English and Arabic. The results are shown in Table 3.

Table 3. Results for All Filtered Objects

\begin{tabular}{|c|c|c|c|c|c|c|}
\hline & \multicolumn{3}{|c|}{ English } & \multicolumn{3}{|c|}{ Arabic } \\
\hline$\frac{\overrightarrow{0}}{\frac{\pi}{0}}$ & 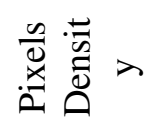 & 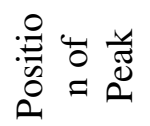 & 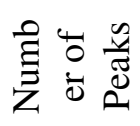 & 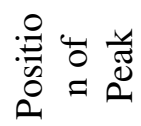 & 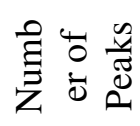 & 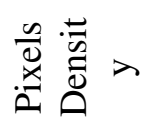 \\
\hline 1 & 8 & 1 & 1 & 0 & 999 & 0 \\
\hline 2 & 3 & 5 & 2 & 5 & 4 & 999 \\
\hline 3 & 2 & 7 & 4 & 3 & 3 & 3 \\
\hline 4 & 7 & 7 & 7 & 3 & 5 & 6 \\
\hline 5 & 2 & 999 & 1 & 7 & 7 & 7 \\
\hline 6 & 9 & 999 & 9 & 999 & 4 & 0 \\
\hline 7 & 3 & 1 & 1 & 9 & 9 & 9 \\
\hline 8 & 4 & 999 & 1 & 4 & 8 & 8 \\
\hline 9 & 1 & 999 & 0 & 5 & 5 & 5 \\
\hline 10 & 5 & 5 & 5 & 5 & 4 & 2 \\
\hline 11 & 9 & 1 & 1 & 999 & 8 & 6 \\
\hline 12 & 2 & 2 & 9 & 2 & 4 & 3 \\
\hline 13 & 5 & 2 & 6 & 2 & 4 & 2 \\
\hline 14 & 8 & 1 & 1 & 0 & $\begin{array}{l}999 \\
\end{array}$ & 7 \\
\hline 15 & 1 & 999 & 999 & 5 & 7 & 5 \\
\hline 16 & 8 & 1 & 1 & 0 & 999 & 0 \\
\hline 17 & 8 & 4 & 4 & 6 & 3 & 2 \\
\hline 18 & 8 & 1 & 1 & 0 & 999 & 1 \\
\hline 19 & 2 & 999 & 1 & 999 & 999 & 7 \\
\hline 20 & 8 & 1 & 1 & 0 & 999 & 0 \\
\hline 21 & 8 & 2 & 0 & 2 & 2 & 0 \\
\hline 22 & 8 & 1 & 1 & 1 & 999 & 0 \\
\hline
\end{tabular}

Number 999 is used for cases when the object was not recognized as a correct number. The total number of objects for the plate of Figure 3 is 22 objects. Each row of Table 3 contains the results of tests for one object. The first entry in Table 3 (under Pixel Density) for Object 1 indicates that the pixel-density algorithm was applied to object 1 and it resulted in recognizing object 1 as the number 8. Position of Peaks and Number of Peaks both returned a 1 for Object 1 . Of course these three algorithms were run on the assumption that Object 1 is an English number. Table 2 shows 
The International Journal of Multimedia \& Its Applications (IJMA) Vol.4, No.5, October 2012

that Objects 2 and 3 are vertically aligned, so looking at the results from all 3 algorithms applied to objects 2 and 3 , we conclude that it must be the number 3 since all three algorithms when we assumed that the object 3 is an Arabic number returned 3. Objects 4 and 5 are vertically aligned and they both agree on being the number 7. Objects 6 and 7 also agree that they are the number 9 (only one algorithm (Position of Peak) was not able to recognize object 6 as an English number. Objects 9 and 10 are also aligned vertically and they agree that they are the number 5 . Objects 12 and 13 are also vertically aligned and they almost agree that they are the number 2 . All other vertically aligned objects do not agree on one number. All other vertically aligned objects do not agree on one number. For example, objects 14 and 15 are vertically aligned with object 14 being below object 15. This means that object 14 is an English character and object 15 is an Arabic Character. Looking at the first three columns of Table S3, in the row corresponding to object 14, two out of three methods agree that object 14 is the English number 1. Compare this result with the last three columns in Table 4 in the row corresponding to object 15. Two out of three methods agree that object 15 is the Arabic number 5. However, since objects 14 and 15 are vertically aligned and the English result does not agree with the Arabic result, then objects 14 and 15 are not considered to be numbers belonging to a Saudi license plate. However, we are not yet in a position to rule out objects 14 and 15 as being part of a Saudi license plate. Objects 14 and 15 can still be letters rather than numbers. The same analysis was done to objects 20 and 21 which are vertically aligned. Object 20 can be recognized as the English number 1 while object 21 can be recognized as the Arabic number 2. This means that the Arabic results do not agree with the English results and therefore we can conclude that objects 20 and 21are not numbers in a Saudi license plate. They can still be letters in the Saudi license plate. Objects 16 and 17 are also vertically aligned. Object 16 is recognized as Arabic 0 while object 17 is recognized as English 4. So English and Arabic results do not agree and therefore objects 16 and 17 are not numbers in the Saudi License plate. Table 4 shows the final resulting number portion of the simulated plate so far.

Table 4. Resulting Numbers for the Plate

\begin{tabular}{|l|l|l|l|l|l|l|l|}
\hline \multicolumn{7}{|c|}{ All Resulting Numbers } \\
\hline 3 & 7 & 9 & 5 & 2 & $?$ & $?$ & $?$ \\
\hline
\end{tabular}

Similarly, the objects are checked to see if they are possible letters. All three algorithms associated with recognizing letters in English and Arabic are run on each object. Tables 5 and 6 show the corresponding results.

Table 5. Results of Letters for All Filtered Objects

\begin{tabular}{|c|c|c|c|c|c|c|}
\hline & \multicolumn{3}{|c|}{ English } & \multicolumn{3}{|c|}{ Arabic } \\
\hline 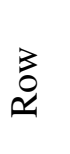 & 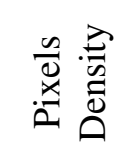 & 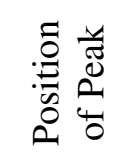 & 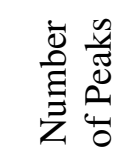 & 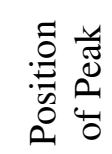 & 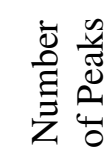 & 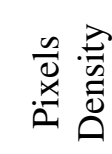 \\
\hline 1 & $\mathrm{~K}$ & $X$ & $\mathrm{~T}$ & $\mathrm{~A}$ & $\mathrm{~A}$ & $\mathrm{H}$ \\
\hline 2 & $\mathrm{~J}$ & B & $\mathrm{S}$ & $\mathrm{U}$ & $\mathrm{E}$ & $\mathrm{Z}$ \\
\hline 3 & $\mathrm{D}$ & $\mathrm{R}$ & $\mathrm{A}$ & A & $\mathrm{U}$ & $\mathrm{E}$ \\
\hline 4 & $\mathrm{~J}$ & $\mathrm{R}$ & A & $\mathrm{F}$ & $\mathrm{H}$ & $\mathrm{K}$ \\
\hline 5 & $\mathrm{~T}$ & $\mathrm{~F}$ & $\mathrm{~T}$ & $\mathrm{~A}$ & $\mathrm{~A}$ & $S$ \\
\hline
\end{tabular}


The International Journal of Multimedia \& Its Applications (IJMA) Vol.4, No.5, October 2012

\begin{tabular}{|r|c|c|c|c|c|c|}
6 & $\mathrm{~V}$ & $\mathrm{~B}$ & $\mathrm{~S}$ & $\mathrm{~V}$ & $\mathrm{E}$ & $\mathrm{V}$ \\
\hline 7 & $\mathrm{~J}$ & $\mathrm{~T}$ & $\mathrm{~T}$ & $\mathrm{Z}$ & $\mathrm{L}$ & $\mathrm{S}$ \\
\hline 8 & $\mathrm{~J}$ & $\mathrm{~J}$ & $\mathrm{U}$ & $\mathrm{K}$ & $\mathrm{X}$ & $\mathrm{K}$ \\
\hline 9 & $\mathrm{X}$ & $\mathrm{R}$ & $\mathrm{D}$ & $\mathrm{N}$ & $\mathrm{S}$ & $\mathrm{D}$ \\
\hline 10 & $\mathrm{~V}$ & $\mathrm{E}$ & $\mathrm{Z}$ & $\mathrm{F}$ & $\mathrm{E}$ & $\mathrm{V}$ \\
\hline 11 & $\mathrm{~J}$ & $\mathrm{~F}$ & $\mathrm{U}$ & $\mathrm{A}$ & $\mathrm{X}$ & $\mathrm{V}$ \\
\hline 12 & $\mathrm{D}$ & $\mathrm{D}$ & $\mathrm{S}$ & $\mathrm{E}$ & $\mathrm{E}$ & $\mathrm{E}$ \\
\hline 13 & $\mathrm{E}$ & $\mathrm{E}$ & $\mathrm{E}$ & $\mathrm{F}$ & $\mathrm{E}$ & $\mathrm{X}$ \\
\hline 14 & $\mathrm{~T}$ & $\mathrm{X}$ & $\mathrm{V}$ & $\mathrm{F}$ & $\mathrm{X}$ & $\mathrm{X}$ \\
\hline 15 & $\mathrm{X}$ & $\mathrm{X}$ & $\mathrm{X}$ & $\mathrm{F}$ & $\mathrm{L}$ & $\mathrm{E}$ \\
\hline 16 & $\mathrm{~K}$ & $\mathrm{X}$ & $\mathrm{F}$ & $\mathrm{A}$ & $\mathrm{A}$ & $\mathrm{H}$ \\
\hline 17 & $\mathrm{~A}$ & $\mathrm{~A}$ & $\mathrm{~A}$ & $\mathrm{~B}$ & $\mathrm{U}$ & $\mathrm{H}$ \\
\hline 18 & $\mathrm{~T}$ & $\mathrm{X}$ & $\mathrm{T}$ & $\mathrm{A}$ & $\mathrm{A}$ & $\mathrm{U}$ \\
\hline 19 & $\mathrm{~F}$ & $\mathrm{X}$ & $\mathrm{T}$ & $\mathrm{V}$ & $\mathrm{F}$ & $\mathrm{S}$ \\
\hline 20 & $\mathrm{~B}$ & $\mathrm{X}$ & $\mathrm{T}$ & $\mathrm{A}$ & $\mathrm{A}$ & $\mathrm{H}$ \\
\hline 21 & $\mathrm{~B}$ & $\mathrm{R}$ & $\mathrm{G}$ & $\mathrm{F}$ & $\mathrm{V}$ & $\mathrm{H}$ \\
\hline 22 & $\mathrm{~F}$ & $\mathrm{~T}$ & $\mathrm{H}$ & $\mathrm{A}$ & $\mathrm{A}$ & $\mathrm{F}$ \\
\hline
\end{tabular}

Table 6. Resultant Possible Letters for the Plate

\begin{tabular}{|l|l|l|l|l|l|l|l|}
\hline$?$ & $\mathrm{~A}$ & $?$ & $?$ & $\mathrm{E}$ & $\mathrm{X}$ & $\mathrm{A}$ & $\mathrm{A}$ \\
\hline
\end{tabular}

The fifth cell in Table 6 shows the grouping of rows 12 and 13 from Table 5. In this case, the most repeated letter is $E$, which is the first letter of the plate of Figure 3. Tables 4 and 6 agree that there are 7 characters that could possibly be a new Saudi license plate. Since in Saudi license plates we have exactly three letters to the right of up to four numbers, this means that the first four characters in Tables 4 and 6 are numbers and the next three characters are letters. The fifth cell in Table 6 shows the grouping of rows 12 and 13 from Table 5. In this case, the most repeated letter is $E$, which is the first letter of the plate of Figure 3. Table 7 shows the final resulting number and letter portions of the plate under consideration.

Table 7. Final License Plate Results

\begin{tabular}{|l|l|l|l|l|l|l|}
\hline 3 & 7 & 9 & 5 & $\mathrm{E}$ & $\mathrm{X}$ & $\mathrm{A}$ \\
\hline
\end{tabular}

\section{ANALYSIS AND RESULTS}

There are three types of thresholds used for the simulation and have to be taken into account for the proposed algorithm. The first one is for the pre-processing stage, when converting the image into its negative and trying to remove most of unnecessary objects. Figure 8 shows a binarized image with a bad thresholding level. No license plate can be located in this figure even by a human being. 
The International Journal of Multimedia \& Its Applications (IJMA) Vol.4, No.5, October 2012

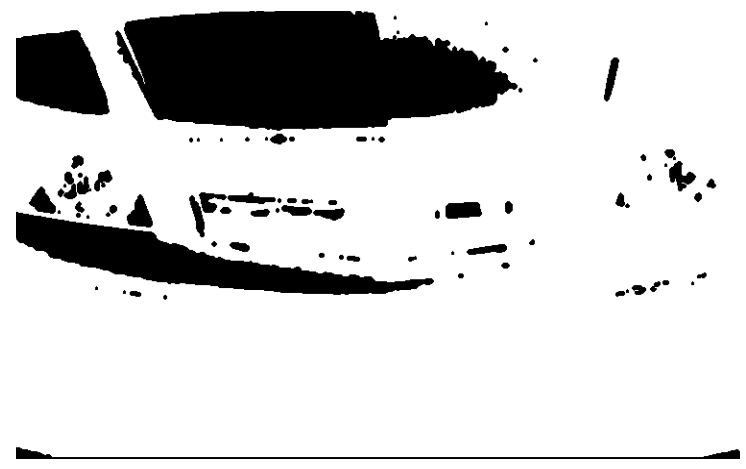

Figure 8: Bad Binarization Threshold

The same figure binarized with an appropriate threshold is shown in Figure 9 which clearly contains a license plate that can be localized and its characters extracted and identified.

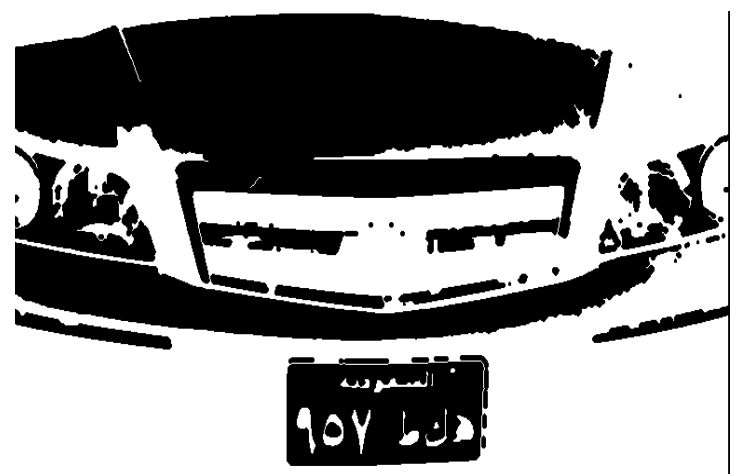

Figure 9: Appropriate Binarization Threshold

The second threshold is before calculating the x-coordinates of the simulated objects, and it is used to remove the rest of the large or small objects that cannot be of any interest as a license plate character. The third type of threshold is when checking the alignments of the objects using their x-coordinates This threshold is used to decide whether two objects are vertically aligned or not.

The algorithm was run on images of 22 plates for a total number of 308 Arabic and English characters. Results show $83.9 \%$ accuracy for identifying the license plates when the size of the plate is small compared to the size of the image as shown in Figure 9a. The accuracy rises to $87.75 \%$ when the size of the plate is comparable to the size of the image as shown in Figure $9 \mathrm{~b}$.

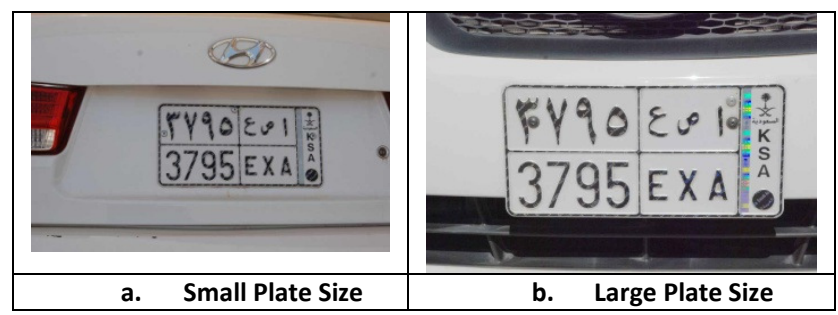

Figure 9: Plate size in an Image 
The International Journal of Multimedia \& Its Applications (IJMA) Vol.4, No.5, October 2012

Two main issues degraded the accuracy of the method. The large screws that are used to mount the plate onto the car might overlap with the characters of the plate. Moreover the rotation of the image of the plate about the y-axis makes it hard for the recognition algorithm to recognize the characters.

\section{CONCLUSION}

In this paper, an algorithm to locate and recognize the characters in an image of a Saudi license plate was presented. Localization of the plate is done by first determining all objects in the image that can possibly be characters in the license plate. Then all large or small objects are discarded and this method concentrates on the objects that have appropriate size. The coordinates of the center point of the bounding box for all remaining objects are found and then possible alignments between these objects are checked. After finding the aligned objects, the recognition algorithms are applied to distinguish between these objects and to differentiate the numbers from the letters in the plate.

The algorithm has shown good results in locating Saudi plates. The only limitations occur when the license plate has large screws or when the license plate is rotated in the image. This algorithm may be adapted to work on non-Saudi license plates since it relies on geometric relationships between objects and the actual shapes of the characters in a license plate. In fact, the algorithm was adapted from an earlier one [16] which was used to locate Lebanese license plates in images.

Future research should focus on automating the thresholds so that they would be function of the image properties rather than fixed for every image. Also the algorithm can be adapted to work on old Saudi license plates if needed by exploiting the horizontal alignment of characters in Saudi license plates rather than only their vertical alignment relationship. Ultimately, the system should be integrated into a full security system along with other recognition algorithms[17].

\section{REFERENCES}

[1] Wenjing Jia, Xiangjian He and Massimo Piccardi, "Automatic License Plate Recognition: a Review," Proceedings of the International Conference on Imaging Science, Systems and Technology, CISST 2004: pp. 43-49.

[2] K. AlMustafa, R. Zantout, H. Obeid, "Peak Position, Recognizing Characters in Saudi License Plates", Accepted for publication, 2011 IEEE GCC Conference and Exhibition for Sustainable Ubiquitous Technology, Dubai, United Arab Emirates, February 19-22, 2011.

[3] Khaled Al Mustafa, Rached Zantout, and Hasan Obeid, "Pixel Density: Recognizing Characters in a Saudi License Plate", the 2010 International Conference on Intelligent Systems Design and Applications (ISDA 2010), Cairo, Egypt, November 29 - December 10, 2010.

[4] K. AlMustafa, R. Zantout, H. Obeid, "Recognizing Characters in Saudi License Plates Using Character Boundaries", International Conference on Innovations in Information Technology (IIT), April 2011, pp. 415 - 420.

[5] Remus Brad, "License Plate Recognition System," Proceedings of the 3rd International Conference on Information, Communications and Signal Processing, Singapore, October 2001.

[6] Serkan Ozbay and Ergun Ercelebi, "Automatic Vehicle Identification by Plate Recognition", World Academy of Science, Engineering and Technology, 2005, pp. 222-225. 
The International Journal of Multimedia \& Its Applications (IJMA) Vol.4, No.5, October 2012

[7] Halina Kwasnicka and Bartosz Wawrzyniak, "License Plate Localization and Recognition in Camera Pictures", Artificial Intelligence Methods, November 13-15, 2002, Gliwice, Poland.

[8] J.R. Parker, Pavol Federl , “An Approach To Licence Plate Recognition”, Vision Interface '97, Kelowna, British Columbia, Canada, May 20-22, 1997.

[9] V. Turchenko, V. Kochan, V. Koval, A. Sachenko and G. Markowsky, "Smart Vehicle Screening System Using Artificial Intelligence Methods," Proceedings of 2003 Spring IEEE Conference on Technologies for Homeland Security, May 7-8, 2003, Cambridge, MA, pp. 182-185.

[10] Jing-Ming Guo and Yun-Fu Liu, "License Plate Localization and Character Segmentation With Feedback Self-Learning and Hybrid Binarization Techniques", IEEE Transactions on Vehicular Technology, vol. 57, no. 3, May 2008.

[11] Hasan Obeid and Rached Zantout, "Line Processing: An Approach to ALPR Character Recognition", ACS/IEEE International Conference on Computer Systems and Applications, Amman, Jordan, May 13-16, 2007.

[12] YUAN Jie, DU Si-dan and ZHU Xian, "Fast Super-resolution for License Plate Image Reconstruction", 19th International Conference on Pattern Recognition, p 1-4, Dec 2008.

[13] K. V. Suresh, G. Mahesh Kumar, and A. N. Rajagopalan, "Superresolution of License Plates in Real Traffic Videos", IEEE TRANSACTIONS ON INTELLIGENT TRANSPORTATION SYSTEMS, VOL. 8, NO. 2, JUNE 2007.

[14] Rami Al-Hmouz - Subhash Challa, "License plate localization based on a probabilistic model", Machine Vision and Applications, 21, p.319-330, 2010.

[15] Rafael C. Gonzalez and Richard E. Woods, "Digital Image Processing," Second edition, Prentice Hall, pp. 523-532, 2002, ISBN: 0130946508.

[16] Hassan Obeid, Rached Zantout and Fadi Sibai, "License Plate Localization in ALPR Systems", 4th International conference on Innovations in Information Technology (Innovations 07), Dubai, United Arab Emirates, November 18-20, 2007.

[17] Aruni Singh, Sanjay Kumar Singh, Shrikant Tiwari, "Comparison of face Recognition Algorithms on Dummy Faces", The International Journal of Multimedia \& Its Applications (IJMA) Vol.4, No.4, August 2012, pp. 121-135. 
The International Journal of Multimedia \& Its Applications (IJMA) Vol.4, No.5, October 2012

\section{Authors}

Dr. Lama Hamandi received her B.E. degree in Electrical Engineering from the American University of Beirut in 1988 and her M.S. and Ph.D. degrees from the Ohio State University in 1995 with a major in Computer Engineering and minor in Computer Science. She is currently a Senior Lecturer at the Electrical and Computer Engineering department in the American University of Beirut. Her research interests include Parallel Architecture, Parallel Processing, Digital Image Processing and Natural Language Processing involving the Arabic Language.

Dr. Khaled Almustafa received his B.E.Sc. in Electrical Engineering, M.E.Sc and $\mathrm{Ph} . D$. in Wireless Communication from the University of Western Ontario, London, Ontario, Canada in 2003, 2004 and 2007 respectively. He is currently the Chairman of the Department of Communication and Networks Engineering, College of Engineering at Prince Sultan University (PSU), Riyadh, K.S.A. Also serving as the General Supervisor for the Information Technology and Computer Services Centre (ITCS) at PSU. His research interests include error performance evaluation of MIMO communication systems in partially known channels, adaptive modulation, and Channel Security as well as text recognition models.

Dr. Rached Zantout received his B.E. from The American University of Beirut, Lebanon in 1988, his MSc from the University of Florida in 1990 and Ph.D. from the Ohio State University in 1994, all degrees being in Electrical Engineering. Currently, $\mathrm{He}$ is an Associate professor at the Math and Computer Science Department at Beirut Arab University. His research interests cover Robotics, Artificial Intelligence, and Natural Language Processing. He currently works on developing components for Machine Translation and Natural Language Processing with a special focus on tools related to the Arabic Language. He also has active research in the area of autonomous robot navigation, Computer Vision, Digital Image Processing and Embedded Systems Design.

Hasan Obeid received his B.S. and M.S. in Computer Science from the Hariri Canadian University in 2004, 2006 respectively. He acquired a number of publications related to image processing and in Automatic License Plate Recognition in particular. He joined Zawya in 2007 and He is currently holding a Technical Lead position in web development. Although Hasan has a web development career but he is an automation enthusiast with a passion for image processing.
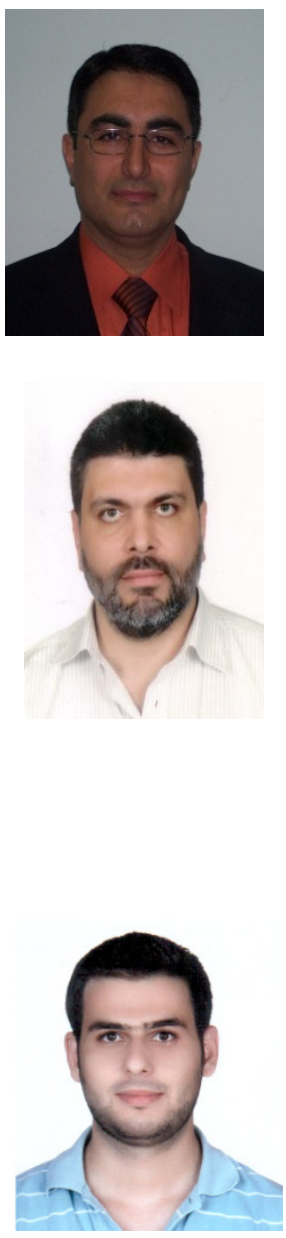